
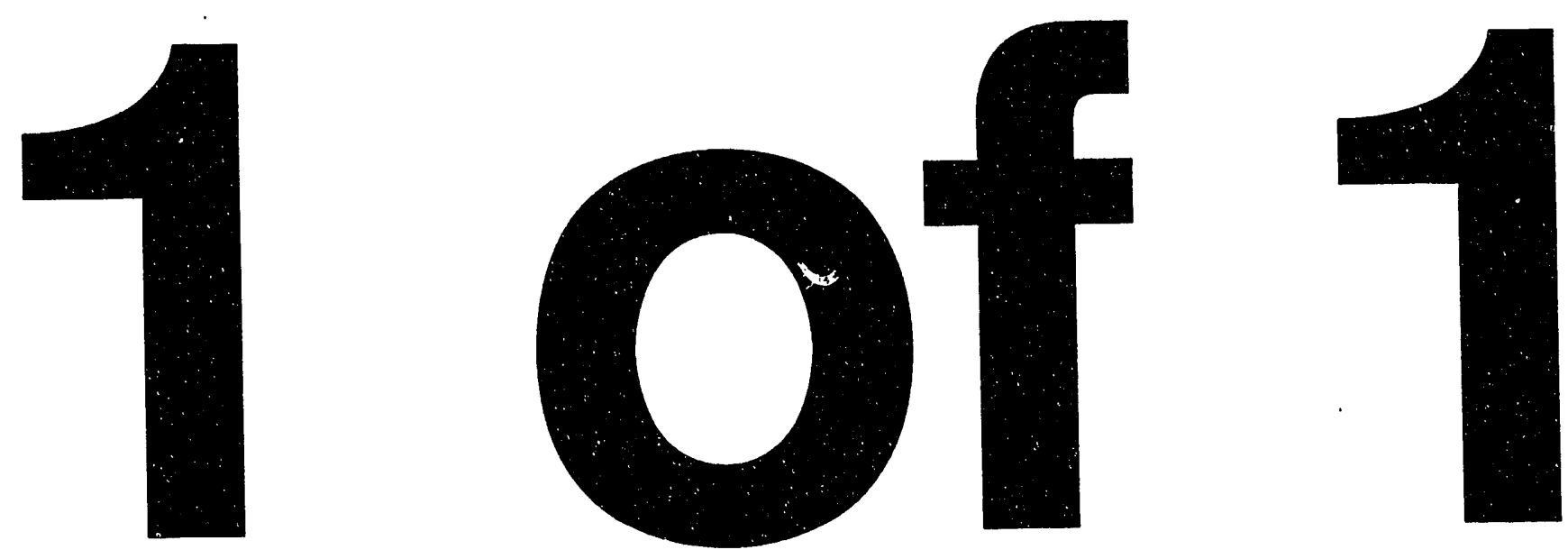


\title{
Preliminary Results from a Study of Collar Lamination Variation in SSC Prototype Dipole Magnets*
}

\author{
R. Gattu, G. Brown, and D. Pollock \\ Superconducting Super Collider Laboratory ${ }^{\dagger}$ \\ 2550 Beckleymeade Ave. \\ Dallas, TX 75237
}

April 1993

\section{MASTER}

*Presented at the Fifth Annual International Symposium on the Super Collider, May 6-8, 1993 San Francisco, CA.

${ }^{\dagger}$ Operated by the Universities Research Association, Inc., for the U.S. Department of Energy under Contract No. DE-AC35-89ER40486. 


\title{
PRELIMINARY RESULTS FROM A STUDY OF COLLAR LAMINATION VARIATION IN SSC PROTOTYPE DIPOLE MAGNETS
}

\author{
R. Gattu, G. M. Brown and D. Pollock \\ Superconducting Super Collider Laboratory* \\ 2550 Beckleymeade Avenue \\ Dallas, TX 75237
}

\section{INTRODUCTION}

The collar laminations used in SSC Prototype Collider Dipole Magnets determine the volume within which the magnet coils are constrained after collaring and keying. The uniformity and symmetry of the inside volume of the collars along the length of the magin: may have a significant influence on the field quality of the finished assembly. This paper describes an on-going Statistical Quality Control study of collar lamination dimensional variation being performed by SSCL Magnet Systerss Division Quality Assurance (MSD QA).

Samples of collars have been measured using a coordinate measuring machine to evaluate manufacturing process capability as well as the overall uniformity of the inventory population of collar laminations. The collar data will be used to predict variation in the coil assembly center and radius for inner and outer top-bottom, left-right coil combinatiors as well as pole angles. Collar results will be combined with azimuthal coil size measuremerits as part of a manufacturing cause and effect model for predicting axial geometric multipoles based on the observed mechanical variation.

This work focuses on Prototype Collider Dipole Magnet DCA102 currently being built at the SSCL MDL in Waxahachie, Texas. This magnet is being made on the same coil curing and collaring mold cavities that were used for the DCA 300 series magnets built at FNAL in 1991-92 and which were later used in the 1992 Accelerator Systems String Test (ASST). The collars are part of the same procurement used for the DCA300 series magnets.

\section{SAMPLING FROM INVENTORY}

Collar laminations have been collected randomly from the SSCL inventory. Ten boxes were selected randomly from inventory for each collar type (left and right hand lamination pairs). From each box of one hundred collars, a sample of ten collars has been collected randomly. Each sample has been identified by a box number and a reference number for measurements.

\section{MEASUREMENT FEATURES}

Critical dimensions which influence the coil to collar and collar to yoke interface features have been measured. The important measured features are collar lamination outer radius, lower inner radius, upper inner radius and pole face angles. Each feature has been measured

\footnotetext{
* Operated by the Universities Research Association, Inc., for the U.S. Department of Energy under Contract NNo. DE-AC.35-89ER4048́
} 
at different locations (left to right for radii and top to botton for pole face angle features) to obtain feature statistics. Each measurement is identified by a ten digit feature number, angular location for radius feature and X Y coordinates for pole face angle features. A total of fifty three individual measurements have been made on each of two hundred collar laminations in the study.

\section{MEASUREMENT PROCEDURE}

Collar laminations have been measured using a Sheffield CORDAX 1808M-DCC (Direct Computer Control) Coordinate Measuring Machine (CMM) at the SSCL. MSD QA Inspection \& Test Group developed a part program to measure collar laminations on the CMM. Collar laminations are mounted for measurement on a specially designed fixture, which can hold ten laminations for each setup. Initially the outer radius feature is measured at eight different locations and the average value is computed to determine the center for Outer Radius (O4R) feature. From the computed center point for O4R feature, the origin is computed and located as per the drawing ${ }^{1}$ requirements and used as a starting point for measurement of additional features. Inner and outer radii features are measured at different horizontal and vertical offsets according to the drawing requirements. Fig 1 illustrates an Inner Left Radius (IL1R) feature measured at four different angular locations.

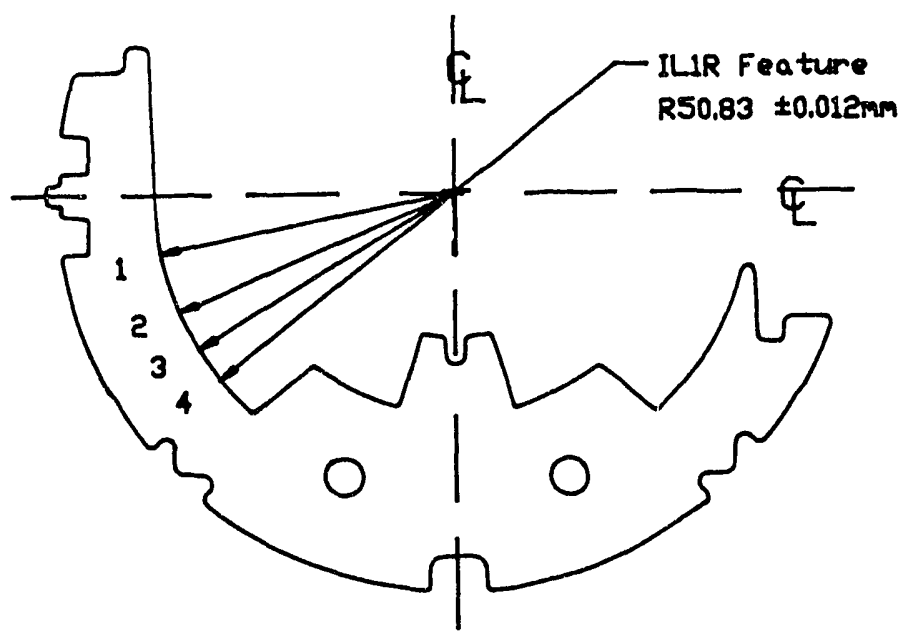

Figure 1. Collider Dipole Magnet Collar Lamination

\section{MEASUREMENT ERROR}

Measurement error studies have been conducted to ensure adequate gage and inspection system capability.

Table 1. Machine Repeatability Measurement Analysis

\begin{tabular}{|c|c|c|c|}
\hline $\begin{array}{c}\text { Lamination } \\
\text { Feature }\end{array}$ & $\begin{array}{c}\sigma \text { gage repeatability } \\
(\mathrm{mm})\end{array}$ & $\begin{array}{c}1^{*} \sigma \text { gage repeatability } / \\
\text { Tolerance } \\
(\mathrm{mm})\end{array}$ & $\begin{array}{c}\left(1^{*} \sigma \text { gage repeatability }\right. \\
/ \text { Tolerance)*100 } \\
(\%)\end{array}$ \\
\hline IL1R01 & 0.00232 & 0.193 & 19.3 \\
\hline IL1R02 & 0.00092 & 0.076 & 7.6 \\
\hline IL1R03 & 0.00105 & 0.087 & 8.7 \\
\hline L1R04 & 0.00108 & 0.090 & 9.0 \\
\hline
\end{tabular}

To evaluate measurement error, one collar lamination was repeatedly measured for all the features ten times. Four locations for IL1R feature have been selected for comparative study to evaluate the measurement error ( $\sigma$ gage repeatability). Table 1 shows the measurement error (repeatability) values for the four locations IL1R01 - 04. The manufacturer's specified repeatability range is $0.003 \mathrm{~mm}\left(0.00012^{\prime \prime}\right)$. The drawing tolerance 
is $\pm 0.012 \mathrm{~mm}$. One $\sigma$ gage repeatability /tolerance ratio is shown for all four locations measured. Repeatability is typically less than ten percent of the part rolerance.

Current calibration 2 of CMM shows that the accuracy and the repeatability values meet the manufacturer's specifications.

\section{PRELIMINARY DATA ANALYSIS}

Measurement data have been obtained for two hundred sampled laminations. Due to space limitations in this paper, only four features (IL1R, Inner lower Radius (I1R), Inner middle Radius (I2R) and O4R) are described for the collars fiom one box sampled. The feature IL1R is illustrated in figure 1 . Graphs and analysis of lamination measurements presented in this paper represent only an initial methodology for describing feature variation. Future studies will analyze the data in detail. Data will be analyzed by two methods, one based on the ideal center (i.e, in accordance with the drawing) and the other based on the best fit center calculated for the part. Both methods will be used to evaluate the shift between the ideal center and the estimated center of the part.

Figure 2 (based on the ideal center measurements) shows variation "within" the lamination and "between" the laminations for the four measurements of the IL1R feature. The two dotted lines in figure 2 represent the drawing lower and upper tolerance limits.

Figure 3 is a box plot for IL1R by measurement. The ideal center data is used for the box plots. The box plot shows an upward trend for the measured position suggesting a systematic deviation in the radius.

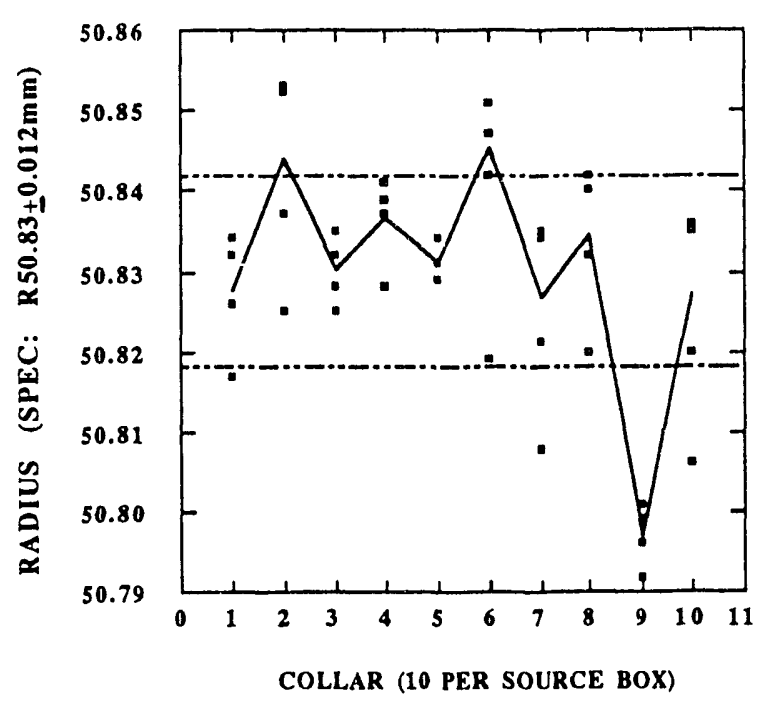

Figure 2. Collar Lamination vs Radius Feature Plot

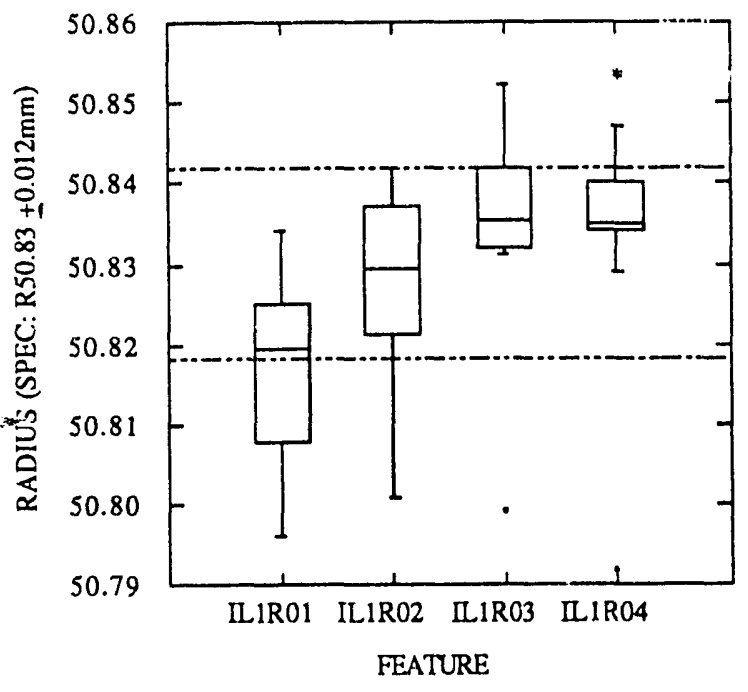

Figure 3. Box Plot for Radius Feature

Figure 4 is a plot based on best fit centers for IIR and I2R features. These features are illustrated in figure 6 . Two vertical and horizontal dotted lines in figure 4 represent the drawing tolerance zones for $\mathrm{X}$ and $\mathrm{Y}$ coordinates for IIR and I2R center points. The enclosed dotted line rectangle is the tolerance zone for the centers of the IIR and I2R features. None of the measured feature centers lie in the drawing specified tolerance zone. These deviations if representative of the inventory population will affect the coil placement in the collared coil. Such deviation in collar geometry is expected to influence field quality ${ }^{3}$.

Figure 5 is a plot based on best fit centers for Outside Radius (O4R) feature. The feature is illustrated in figure 6. Best fit center points for O4R feature are lying in the drawing specified tolerance zones for all the laminations except one. Similar comparisons will be studied for other outer surfaces $(\mathrm{O} 4 \mathrm{R})$ to evaluate collar influence on yoke collar interface. 


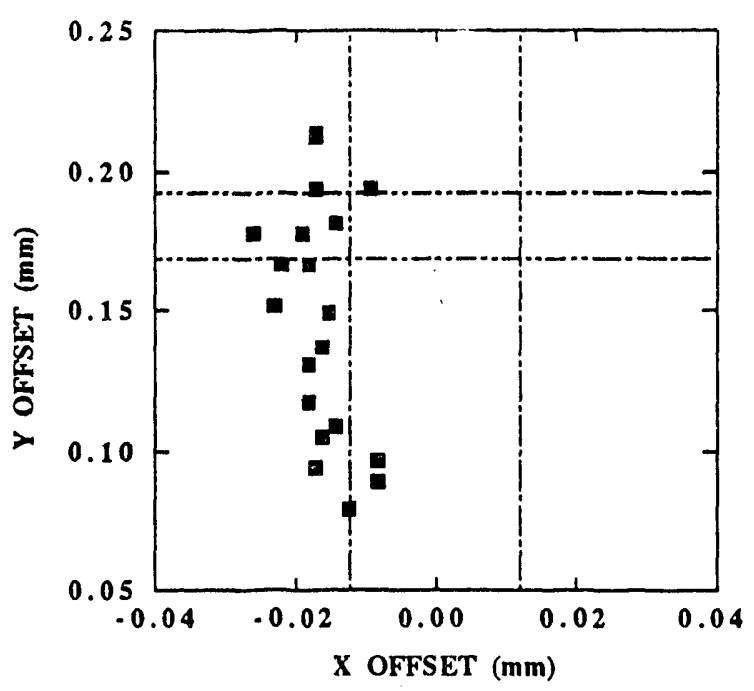

Figure 4. Collar Inner Radius Center (IIR AND I2R) Feature

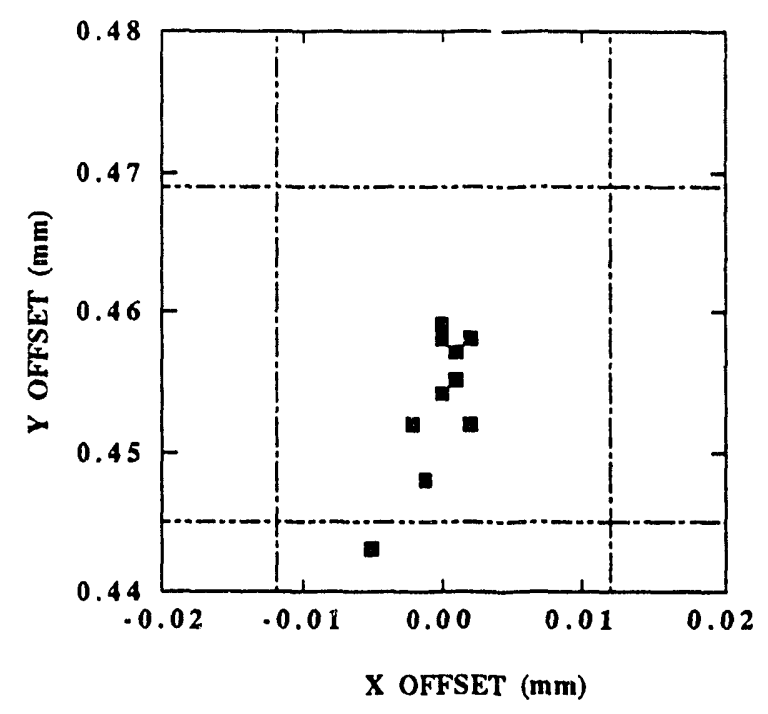

Figure 5. Collar Outer Radius Center (O4R) Feature

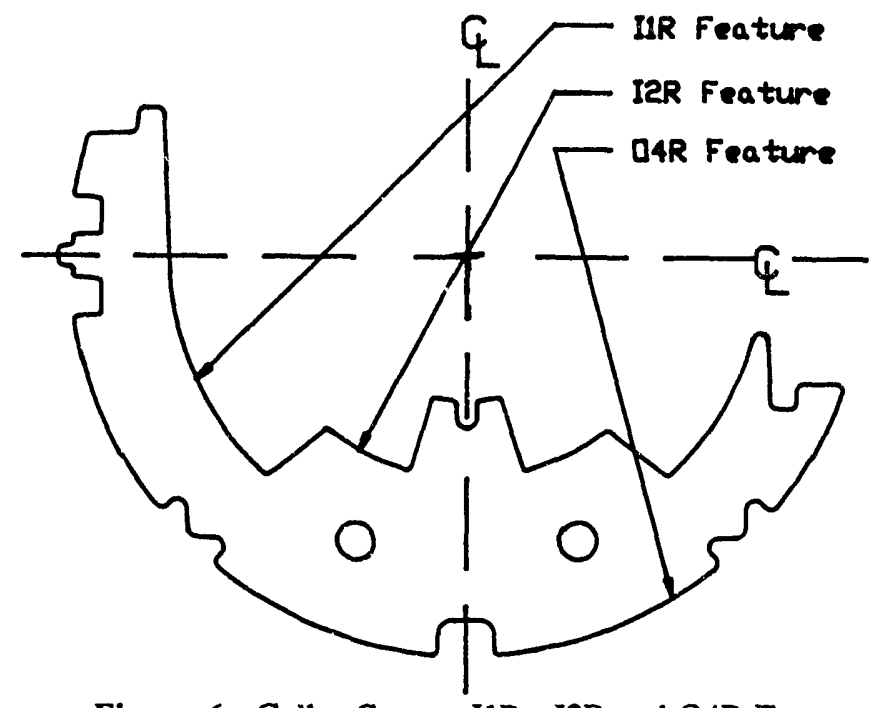

Figure 6. Collar Centers IIR, I2R and O4R Features

\section{CONCLUSIONS}

We have described a set of prototype dipole collar lamination measurements taken as part of a statistical quality control study of critical component manufacturing variation. As a result of collecting comprehensive set of measurements from a sample of collars in our inventory, we are now able to study the level of agreement between drawing requirements and manufactured parts in great detail. Collar manufacturing process capability for the source supplier can also be estimated from the compiled results. By mapping the location of the source boxes of sampled laminations, as placed in DCA102, we plan to study the correlation between collar dimensional variation and magnetic field quality.

\section{REFERENCES}

1. FNAL Drawing \# 0102-ME- 292059, Revision C, SSC 50mm Dipole Collar Lamination For Vertically Split Yoke.

2. CMM Certificate of Calibration, SSCL MSD QA (Inspection \& Test Group), January 1993.

3. Document Number MD-MRA-A-93-001, Requirements and Specifications for SSC $50 \mathrm{~mm}$ Collider Dipole Magnet, Section 2.3.3.6, August 1991. 

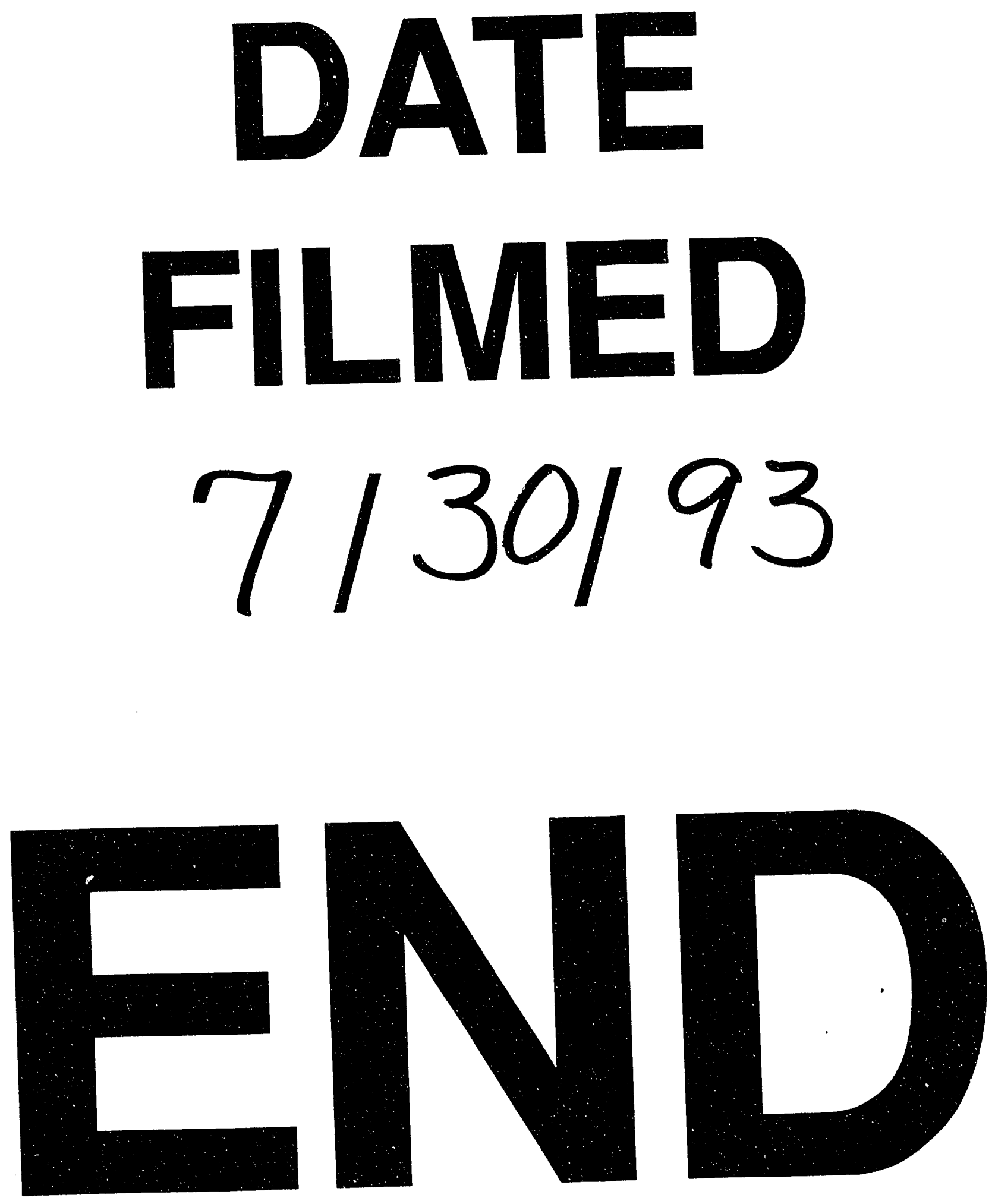

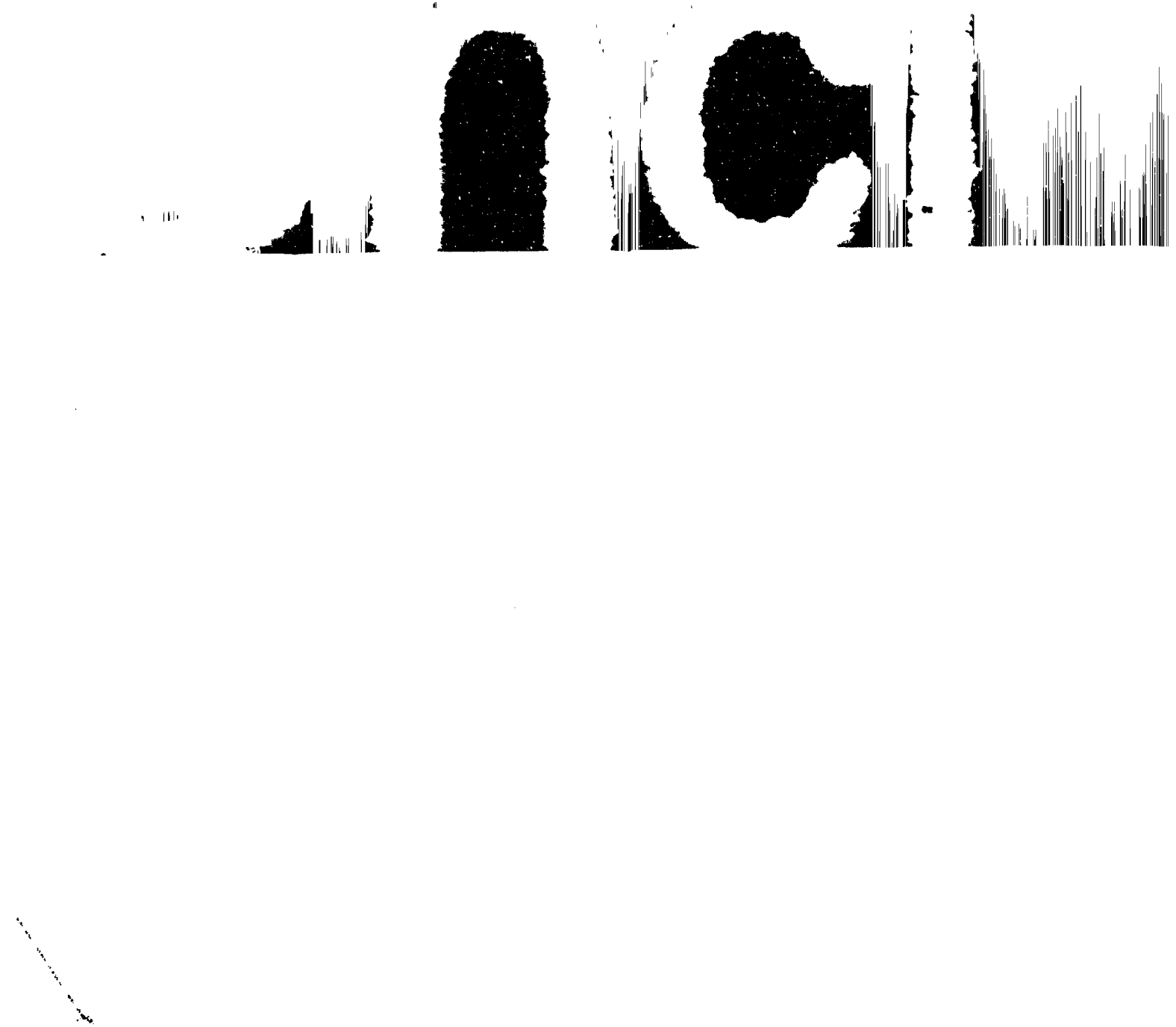